\title{
Erratum: Cinderella versus the Wicked Stepmother
}

Marijke Hans L. Bodlaender ${ }^{1}$, Cor A.J. Hurkens ${ }^{2}$, Vincent J.J. Kusters ${ }^{2}$, Frank Staals ${ }^{2}$, Gerhard J. Woeginger ${ }^{2}$, and Hans Zantema ${ }^{2}$

${ }^{1}$ Dept. of Information and Computing Sciences, Universiteit Utrecht, Netherlands

${ }^{2}$ Dept. of Mathematics and Computer Science, TU Eindhoven, Netherlands

DOI 10.1007/978-3-642-33475-7_28

The name of the first author of the paper starting on page 57 of this volume has been printed incorrectly. It should read: Marijke H.L. Bodlaender 